\title{
Minimum alveolar concentration: A reconsideration
}

\author{
Tijun Dai, MD ${ }^{1 *}$, Wei Cheng, $\mathrm{MD}^{2}$, Qin Yin, $\mathrm{MD}^{2}$, He Liu, MD, PhD ${ }^{2,3}$, Yi Lyu MD ${ }^{4}$
}

${ }^{1}$ Jiangsu Province Key Laboratory of Anesthesia and Analgesia Application Technology \& Jiangsu Province Key Laboratory of Anesthesiology, Xuzhou Medical University; ${ }^{2}$ Department of Anesthesiology, The Affiliated Hospital of Xuzhou Medical University; ${ }^{3}$ Department of Biology, New York University Abu Dhabi, Abu Dhabi 129188, United Arab Emirates; ${ }^{4}$ Department of Medical Affairs, Techpool Bio-Pharma Co.,Ltd., Guangzhou

\begin{abstract}
Minimum alveolar concentration (MAC), served as the vital concept for standard measure of potency for inhaled volatile anesthetic agents, has been employed in anesthetic practice for more than 50 years. A re-consideration in the connotation and influence factors on MAC is introduced in this article. MAC actually indicates "median effective dose (ED50) for immobility", but it is at least not comprehensive for MAC being as anesthetic potency, the total effects of inhaled volatile anesthetic agents. Without a strict "additive" nature, MAC is only the potency rather than efficacy, and the measured value of MAC usually is more than that of ED50 for the disappearance of righting reflex. Some factors, such as duration time of anesthesia, and the pattern, intensity and positions of stimulus, all can affect the measured value of MAC. In the future, in order to make MAC play more important and scientific role in the anesthetic practice, there should be an intensive study on the measured value of MAC of inhaled volatile anesthetic agents in different situations including the pattern, intensity and location of the stimulus.
\end{abstract}

Keywords: minimum alveolar concentration; median effective dose; potency; efficacy; immobility; muscular relaxation; analgesia

\section{Introduction}

Minimum alveolar concentration (MAC) provides a target-controlled titration and real-time feedback of inhaled volatile anesthetic agent administration. It is defined as the minimum alveolar concentration at sea level of inhaled anesthetic required to prevent apparently purposeful movement in $50 \%$ of patients in response to a "standardized" surgical incision under a standard atmospheric pressure. [1]
MAC served as the vital standard measure of potency for inhaled volatile anesthetic agents and has been employed in anesthetic practice for more than 50 years, since Eger et al. introduced the concept in 1965. [2] Nevertheless, it so far has had and will still have great significance in the future in quantitative effect comparison and guiding the clinical anesthesia application of different inhaled volatile anesthetic agents. Currently the authors provided some new thinking in the connotation and influence factors on MAC after years of practice and thinking.

\section{MAC indicates "ED50 (median effective dose) for im- mobility"}

The measured value of MAC refers to the measurement of end-tidal anesthetic concentrations of volatile anesthetic agent administration when there is half of the experimental subject immobility in response to a given noxious stimulus, such as clamping, skin incision or electrical stimulation by sequential method (or up-down method). Obviously, MAC is the quantal response or all-or-none response, equivalent to ED50, and matches the end-tidal concentration without movement as an index, so it in fact indicates "ED50 for immobility", the effective dose for $50 \%$ of subjects without movement. In light of individual variation, it is difficult to measure the minimum alveolar concentration, and so ED50 is only used in pharmacology. The ED50 is a single point on the steepest part of dose-response curve, and response will have an obvious change with any slight change in dose. Based on these reasons, it is not appropriate to define MAC as "minimum dose of alveolar concentration". The authors suggest defining the MAC as "median effective dose for immobility" or "ED50 for immobility".

The mobility of body results from pain induced by nociceptive stimulus and the reflex contraction of skeletal muscle. MAC indicates the volatile anesthetic agents have the effect 
Table 1.The measured values MAC of different inhaled anesthetic agents

with and without $70 \%$ nitrous oxide $\left(\mathrm{N}_{2} \mathrm{O}\right)$

\begin{tabular}{|l|c|c|c|c|c|}
\hline & Ether & Halothane & Methoxyflurane & Enflurane & Isoflurane \\
\hline $\mathrm{O}_{2}$ & 1.92 & 0.77 & 0.16 & 1.68 & 1.15 \\
\hline $70 \% \mathrm{~N}_{2} \mathrm{O}$ & 1.0 & 0.29 & 0.07 & 0.57 & 0.5 \\
\hline Ratio & 0.52 & 0.38 & 0.44 & 0.34 & 0.48 \\
\hline Reduction (\%) & 48 & 62 & 56 & 66 & 56 \\
\hline
\end{tabular}

$70 \% \mathrm{~N}_{2} \mathrm{O}: 0.7 / 1.05=0.67 \mathrm{MAC}, 1 \mathrm{MAC}-0.67 \mathrm{MAC}=0.33 \mathrm{MAC}$.

of immobility, which is the combined effects of analgesia and the inability to move, and it is still not clear what the proportion of each role of these two effects is.

\section{MAC does not reflects anesthetic potency}

MAC has long been used as the index of anesthetic potency of volatile anesthetic agents. In fact, MAC only reflects the effect of immobility induced by an agent, rather than the other effects. The less value of MAC means the stronger effect to produce immobility and vice versa. In addition to analgesia, immobility, anesthetic potency of volatile anesthetic agents includes sedative, hypnosis, stability, cognitive dysfunction, consciousness loss, stress reaction inhibition etc. And that, just for analgesia or insensitive to pain, MAC only reflects the response of skin and subcutaneous tissue to nociceptive stimulus, a standardized surgical stimulation (incision). However, patients who underwent operation also are subjected to stimulus from endotracheal intubation, stripping rib, visceral traction, or angiokinesis, etc., which can't be reflected by MAC. [3]

MAC approximately equals to ED50, and different MAC can be obtained by change in the pattern, intensity of stimulus and observed indicator, such as "MAC-intubation", "MAC-awake" and so on. Although MAC as an important concept reflects the most important anesthesia roles of volatile anesthetic agents, it is at least not comprehensive for MAC being as anesthetic potency, the total effects of inhaled volatile anesthetic agents, and the usefulness of an agent. The best example is that metoksyfluran with the smallest value of MAC $(0.16 \%)$ has not been used in clinic, but nitrous oxide with the biggest value of MAC $(105 \%)$ has still been widely used.

\section{MAC as anesthetic potency not efficacy}

Efficacy is defined as the biggest effect of drugs without limitation of dose, and anesthetic efficacy usually refers to the maximum depth of anesthesia. If given enough concentration, ether and halothane can both make patients have three to four stage anesthesia, or even died of medul- la oblongata anesthesia, which is a high efficacy general anesthetic. As for nitrous oxide, even inhaled concentration is as high as $80 \%$, only can cause light anesthesia, and $100 \%$ nitrous oxide is forbidden for use alone to avoid death caused by hypoxia, so nitrous oxide is a low efficacy general anesthetic.

Potency is defined as the required dose or concentration that has effects. MAC described as the concentration of inhaled anesthetic agent required to prevent apparently purposeful movement in 50\% of experimental subjects in response to nociceptive stimulus, is potency not efficacy. Although halothane belongs to the same efficacy of inhaled anesthetic agent, halothane has lower MAC $(0.77 \%)$ comparing with others (MAC 1.92\%), which indicates that halothane has a higher potency. Nitrous oxide, the value of its MAC as high as $105 \%$, has not only weak efficacy but also a low potency. It is the "equivalent concentration" of inhaled anesthetic agents that have the equivalent MAC value, and the comparison among different inhaled anesthetic agents should be made under the condition of the equivalent MAC value, such as 0.7 MAC. Potency and efficacy is often confused in some published literatures, which we have discussed previously. [4]

\section{MAC lack of a strict "additive" nature}

It is generally recognized that MAC have a "additive" nature, means that the same MAC of one inhaled anesthetic agent can replace the other one, for example, the effect of 0.5 MAC halothane plus 0.5 MAC enflurane has the same effect of 1 MAC ether or isoflurane. But in fact, MAC of inhaled anesthetic agents does not have a strict "additive" nature (as shown in Table 1). Some studies even found that there was antagonistic action when nitrous oxide and isoflurane was conjunctively used.[5]

\section{MAC is not the ED50 for "loss of righting reflex"}

Righting reflex is the animal instinct to keep animal standing position. The time of loss of righting reflex is an often used index for hypnosis. The regulation centers of righting 
Table 2. The ratio of MAC to ED50 for "loss of righting reflex"

\begin{tabular}{|c|c|c|c|c|c|c|}
\hline Halothane & Enflurane & Isoflurane & Chloroform & $\begin{array}{c}\text { Cyclopro- } \\
\text { pane }\end{array}$ & $\begin{array}{c}\mathrm{N}_{2} \mathrm{O} \\
\text { Methoxyflu- } \\
\text { rane }\end{array}$ \\
\hline 1.67 & 1.91 & 2.10 & 1.61 & 1.97 & $>1.82$ & $1.63 \sim 2.08$ \\
\hline
\end{tabular}

$\mathrm{N}_{2} \mathrm{O}$; nitrous oxide

reflex locate in the brainstem and superior center of brain, but the analgesic action site of inhaled anesthetic agents is spinal cord. There are different regulation centers, routes, interaction property and dose between MAC and ED50 for "loss of righting reflex", and the ratio of MAC to ED50 for "loss of righting reflex" is rough 1.8, varied in different inhaled anesthetic agents (as shown in Table 2). So when the concept is used, we must not confuse the MAC with ED50 for "loss of righting reflex".

\section{Factors that affect MAC}

Increasing or decreasing the anesthetic concentration required to prevent movement, thereby many factors alter MAC.[6]

\section{Duration of anesthesia}

It was thought that MAC was a constant during the whole anesthesia, indicating duration time of anesthesia does not affect the MAC. Petersen-Felix et al [7] found that the MAC of isoflurane is $1.28 \pm 0.22 \%$ at the beginning of the operation, however, $173 \mathrm{~min}$ after operation it decreased to $1.04 \pm 0.22 \%$ in 10 patients undergone isoflurane anesthesia maintenance, and Zbinden [8] obtained the similar results after a series of studies on different factors that affect MAC. It was confirmed that the concentration of sevoflurane for maintaining immobility and stability of circulation under the condition of skin incision for placing the chest drainage tube, is significant lower than that at the beginning of the operation. [9] These data show that MAC reduced with duration time of anesthesia. It is speculated that the change has relation to variance in individual sensitive to inhaled anesthetic agents, equilibrium time of inhaled anesthetic agents in spinal cord.

\section{Pattern, intensity and location of the stimulus}

The measured value of MAC was thought to be a constant without being affected by pattern of stimulus, if given percutaneous stimulation like tail clamping, skin incision or electrical stimulation. But Zbinden [8] found the measured value of isoflurane MAC under electric pulse stimulation was $1.03 \pm 0.09 \%$, which was lower than that $1.16 \%$ under skin incision. And "MAC-intubation" and "MAC-awake" also is used now in clinic anesthesia practice. Satas et al
[10] found that no matter under the condition of normal temperature or low temperature, the measured value of halothane and isoflurane MAC under tail clamping was lower than that under jaw clamping. All above results suggested that different pattern, intensity and position of stimulus had different MAC, so when MAC is measured, it should be given the uniform pattern, intensity and location of the stimulus.

\section{Other factors}

The temperature of the human body, change of the hydrostatic pressure, age, and change of $\mathrm{Na}^{+}, \mathrm{K}^{+}, \mathrm{Ca}^{2+}, \mathrm{Mg}^{2+}$ in cerebrospinal fluid, [1] can affect MAC of inhaled anesthetic agents in different degree. Savitha KS and his colleagues [11] found that preemptive multimodal analgesia had synergistic effect with isoflurane (ISO), so they suggested reduction of MACISO to skin incision to a greater degree.

\section{Application of MAC}

Despite its limitations, MAC remains the most commonly used measure of anesthetic potency for inhaled medications. MAC is still a pragmatic surrogate for general anesthetics target concentration in brain, an index for comparing the analgesia potency of inhalation anesthetics. However, it is critical to redefine some basic concepts related to MAC to make it to serve basic science and clinical practice in a precise manner.

\section{Disclosure of Funding: None}

Conflict Interests Disclosure: The authors have no conflicting interests to disclose.

Corresponding Author: Tijun Dai, MD, Professor, Jiangsu Province Key Laboratory of Anesthesia and Analgesia Application Technology \& Jiangsu Province Key Laboratory of Anesthesiology, Xuzhou Medical University, No. 209 Tongshan Road, Xuzhou 221004, Jiangsu Province, PR China. E-mail: 15150019359@163.com.

Editor: Renyu Liu, MD; PhD. Associate Professor, 
Department of Anesthesiology and Critical Care; Perelman School of Medicine at the University of Pennsylvania, 336 John Morgan building, 3620 Hamilton Walk, Philadelphia, PA 19104 .Phone: 2157461485; FAX: 2153495078.1iur@ uphs.upenn.edu

\section{Additional publication details}

Journal short name: Transl Perioper \& Pain Med

Received Date: Jan 15, 2017

Accepted Date: March 15, 2017

Published Date: March 22, 2017

Transl Perioper \& Pain Med 2017; 2(2):13-16

\section{Citation and Copyright}

Citation: Dai T, Cheng W, Yin Q, Liu H, Lyu Y. Minimum alveolar concentration: A reconsideration. Transl Perioper \& Pain Med 2017; 2(2): 13-16

Copyright: (C) 2017 Dai T, et al. This is an open-access article distributed under the terms of the Creative Commons Attribution License, which permits unrestricted use, distribution, and reproduction in any medium, provided the original author and source are credited.

\section{References}

1. Perouansky M, Pearce RA, Hemmings HC., Jr. (2010) Inhaled Anesthetics: Mechanisms of Action. In: Miller RD, Eriksson LI, Fleisher LA, Wiener Kronish JP, Young WL, editors. Miller's Anesthesia, 7th ed. Philadelphia, PA: Churchill Livingstone. pp. 515-588.

2. Guedel AE. (1951) Inhalation Anesthesia: a Fundamental Guide. In: Keys TE, editor. The History of Surgical Anesthesia, 2nd ed. New York: Macmillan. pp. 1-36.

3. Pilge S, Jordan D, Kreuzer M, Kochs EF, Schneider G. Burst suppression-MAC and burst suppression-CP as measures of cerebral effects of anaesthetics. Br J Anaesth. 2014;112:1067-1074.

4. Tijun Dai. Efficacy and potency of anesthetics. Journal of Clinical Anesthesia 1995, 11: 85

5. Eger EI 2nd, Tang M, Liao M, Laster MJ, Solt K, Flood P, Jenkins A, Raines D, Hendrickx JF, Shafer SL, Yasumasa T, Sonner JM. Inhaled anesthetics do not combine to produce synergistic effects regarding minimum alveolar anesthetic concentration in rats. Anesth Analg, 2008, 107: 479-485.PMID: 18633026
6. Villafranca A, Thomson IA, Grocott HP, Avidan MS, Kahn S, Jacobsohn E. The impact of bispectral index versus end-tidal anesthetic concentration-guided anesthesia on time to tracheal extubation in fast-track cardiac surgery. Anesth Analg. 2013;116:541-548. PMID: 23400982

7. Petersen-Felix S, Zbinden AM, Fischer M, Thomson DA. Isoflurane minimum alveolar concentration decreases during anesthesia and surgery. Anesthesiology, 1993, 79: 959-965.PMID: 8239014

8. Zbinden AM, Maggiorini M, Petersen-Felix S, Lauber R, Thomson DA, Minder CE. Anesthetic depth defined using multiple noxious stimuli during isoflurane/oxygen anesthesia. I. Motor reactions. Anesthesiology, 1994, 80: 253-260.PMID: 8311307

9. Han WB, Li TY, Song YY, Jin B. The comparation of requirements of sevoflurane for skin lesion in different time points of anesthesia. Chinese Journal of Surgery, 1997, 35: 512 .

10. Satas S, Haaland K, Thoresen M, Steen PA. MAC for halothane and isoflurane during normothermia and hypothermia in the newborn piglet. Acta Anaesthesiol Scand, 1996, 40: 452-456.PMID: 8738690

11. Savitha KS, Dhanpal R, Shilpa J. The effect of multimodal analgesia on minimum alveolar concentration of isoflurane for skin incision at constantbispectral index. Anesth Essays Res. 2016;10:473-477. PMID: 27746535 